\title{
Determinação de metanol em bebidas alcoólicas utilizando colorimetria por imagens digitais
}

\section{Josiane Amorim Reis ${ }^{1}$, Valdomiro Lacerda Martins, ${ }^{1}$, , Wellington da Silva Lyra ${ }^{2}$ e Paulo Roberto Barros Gomes ${ }^{3}$}

\author{
${ }^{1}$ Universidade Federal do Amazonas. Instituto de Ciências Exatas e Tecnologia. \\ Campus Itacoatiara. Rua Nossa Senhora do Rosário, S/№. Tiradentes. \\ Itacoatiara-AM, Brasil (CEP 69103-128).*E-mail: valdomiro@gmail.com. \\ ${ }^{2}$ Universidade Federal da Paraíba, Centro de Ciências Exatas e da Natureza. \\ Campus I. João Pessoa-PB, Brazil (Caixa Postal 5093, CEP 58051-970). \\ ${ }^{3}$ Instituto Federal de Educação, Ciência e Tecnologia do Pará. Campus Abaetetuba. \\ Avenida Rio de Janeiro, 3322. Francilândia. Abaetetuba-PA, Brasil \\ (CEP 68440-000).
}

Resumo. Este trabalho propõe um método colorimétrico por imagens digitais para determinação de metanol em bebidas alcoólicas. O método é baseado na oxidação do metanol a formaldeído, seguido da reação com ácido cromotrópico produzindo um composto púrpura. Imagens digitais do produto de reação são capturadas usando um scanner e as estas são tratadas usando o programa ImageJ. As condições experimentais foram otimizadas e uma curva analítica, usando o índice colorimétrico de imagens digitais como resposta analítica, foi construída na faixa de $0,0100 \%-0,1000 \% \mathrm{v} / \mathrm{v}$ de metanol. Os limites de detecção e quantificação foram $1,95 \times 10^{-3} \%$ e $6,50 \times 10^{-3} \% \mathrm{v} / \mathrm{v}$, respectivamente. A viabilidade do método é ilustrada na aplicação em amostras de cachaça e licor. Em comparação com a espectrometria ultravioleta-visível, os resultados não mostraram diferenças estatisticamente significativas mediante aplicação do teste- $t$ emparelhado ao nível de confiança de 95\%. Em comparação com outros métodos semelhantes, o proposto apresentou uma sensibilidade superior. Tal característica vantajosa é atribuída à natureza trivariada da detecção do scanner.

Palavras-chave: Colorimetria por imagens digitais; Escaner; Microplaca; Metanol; Bebidas alcoólicas.

\section{Abstract. Determination of methanol in alcoholic beverages using digital image colorimetry. This work proposes a digital image colorimetric method for determination of methanol in alcoholic beverages. The method is based on oxidation of methanol to formaldehyde followed by reaction with chromotropic acid yielding a purple product. Digital images of the reaction product are captured by a scanner and the are treated by ImageJ software. Experimental conditions have been optimized}

Recebido

$05 / 05 / 2020$

Aceito

$14 / 06 / 2020$

Disponível on line $15 / 06 / 2020$

Publicado

$31 / 08 / 2020$

Acesso aberto

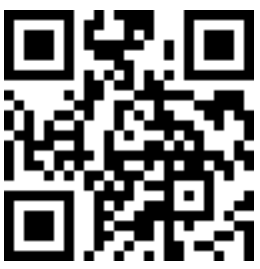

ORCID

(D) 0000-0003-0759-2179 Josiane Amorim Reis

(D) 0000-0001-7362-5050

Valdomiro Lacerda

Martins

0000-0002-3499-6163

Wellington da Silva

Lyra

ISSN 2359-1412/RBGAS-2020-0075/2020/7/16/1/455 
and an aanlytical curve, using digital image colorimetric index as analytical response, was built in the range from $0.0100 \%-0.1000 \% \mathrm{v} / \mathrm{v}$ of methanol. The limits of detection and quantification were $1.95 \times 10^{-3} \%$ and $6.50 \times 10^{-3} \% \mathrm{v} / \mathrm{v}$, respectively. The feasibility of the method is illustrated on the application in cachaça and liquor samples. In comparison with ultraviolet-visible spectrometry, no statistic difference have been observed between the results by applying the paired $t$-test at the 95\% confidence level. In comparison with similar methods, it presented higher sensitivity. Such advantageous characteristic is attributed to the trivariate nature of the detection of the scanner.

Keywords: Digital image colorimetry; Scanner; Microplate; Methanol; Alcoholic beverages.
다 0000-0002-4221-6577 Paulo Roberto Barros Gomes

\section{Introducão}

Análises químicas são realizadas diariamente para determinação de uma variedade de substâncias/íons e a técnica analítica mais comum de realizá-las é por meio da espectrometria de absorção molecular nas regiões ultravioleta-visível (UV-Vis) (Gomes et al., 2008). Utilizando esta técnica analítica para análise quantitativa de muitas amostras, frequentemente são necessárias quantidades grandes de reagentes e tempo de trabalho da ordem de horas até dias. Neste sentido, várias reações colorimétricas padrão foram miniaturizadas para micropoços em microplacas, incluindo métodos úteis para medidas nas áreas da indústria, ciência dos materiais, análises clínicas, biologia, ciências forenses, farmácia, análise de alimentos e estudos ambientais (Soldat et al., 2006).

Considerando aspectos econômicos tais como custo de aquisição e de manutenção de espectrômetros UV-Vis, a análise por imagens digitais (AID) surgiu nas últimas décadas do século passado como uma poderosa e flexível ferramenta analítica alternativa (Paciornik et al., 2006) com as seguintes vantagens:

(1) Permitir ao usuário coletar conjuntos de dados muito maiores e obter resultados estatisticamente confiáveis (Martins et al., 2000).

(2) Simplicidade, baixo custo, frequência analítica e desempenho similar aos instrumentos comerciais (Geladi et al., 1992; Zheng et al., 2004; Prats-Montalbán et al., 2011).

(3) Possibilidade de portabilidade para aplicações in situ (Santos e Pereira-Filho, 2013).

(4) É possivel salvar as imagens em diferentes formatos de arquivo (tiff, bmp, jpeg) e armazená-las por longos períodos de tempo sem afetar a sua qualidade, além de acessá-las instantaneamente para uma eventual reavaliação (Soponar et al., 2008).

A aquisição de imagens digitais pode ser feita por meio de câmeras digitais, câmeras de video (webcams), scanners e smartphones. Devido à iluminação uniforme de superfícies, tempo curto de varredura (10-20 s) e alta resolução óptica, scanners são adequados para registrar imagens digitais de microplacas (Soponar et al., 2008). Entre as técnicas de AID, a colorimetria por imagens digitais (CID) é considerada um novo tipo de técnica já pronta e pode ser interpretada uma colorimetria por reflexão da luz (Lopez-Molinero et al., 2010). Em outras palavras, a luz que alcança o detector de imagem é a luz refletida pelos objetos (Byrne et al., 2000). 
O uso de scanner para métodos para de CID objetivando análise quantitativa já está bem estabelecido na literatura e duas formas de trabalho podem ser reconhecidas:

(1) Análise direta: quando o próprio analito exibe cor.

(2) Análise indireta: quando o analito é convertido (por meio de uma reação química) numa espécie que possui cor. Este processo também é conhecido como derivatização.

No Brasil, a análise de compostos voláteis em bebidas alcoólicas é um parâmetro importante a fim de verificar se as características do produto estão de acordo com a legislação vigente por causa da formação de compostos secundários durante o processo de fermentação (Ferreira e Montes, 1999). A presença de tais compostos é indesejável devido às suas propriedades tóxicas. Um desses compostos é o metanol, que está naturalmente presente em bebidas alcoólicas destiladas e fermentadas (Zacaroni et al., 2011) e é facilmente absorvido pelo organismo, se acumulando no sangue, músculos e olhos (Caruso et al., 2010; Ohimain, 2016). Para o ser humano, o consumo de $20 \mathrm{~mL}$ de metanol pode causar cegueira e o de $60 \mathrm{~mL}$ pode ser mortal sem tratamento adequado (Péres et al., 2012).

Desconsiderando a adição intencional de metanol (adulteração) em bebidas alcoólicas fermentadas e destiladas, sua presença está associada à atividade da enzima pectinase em meio ácido que promove a degradação da pectina, um polissacarídeo que está presente na parede celular vegetal (Seyfried et al., 2016). Como produtos são obtidos moléculas de ácido galacturônico e metanol (Canteri et al., 2012) conforme a Figura 1.
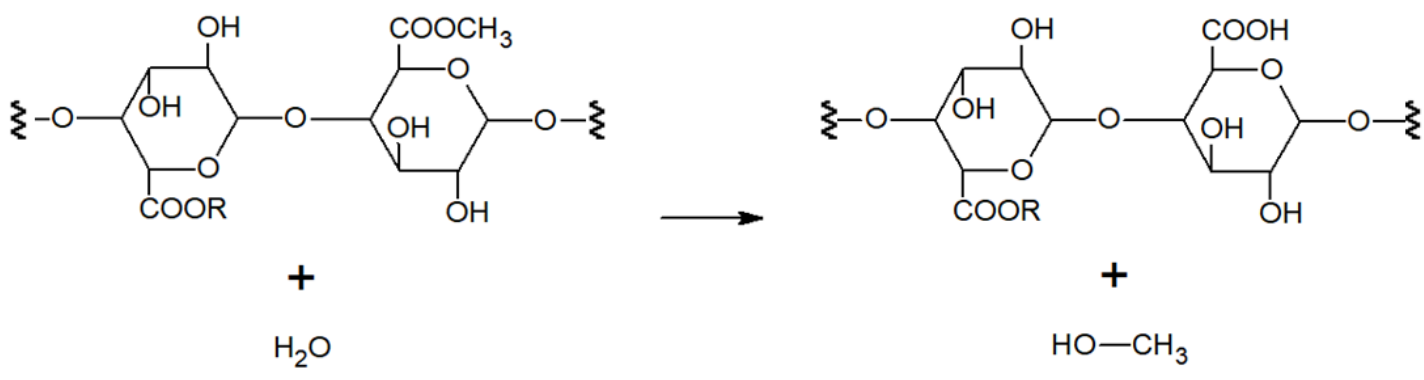

Figura 1. Reação de produção de metanol em bebidas alcoólicas a partir da pectina, mediada pela enzima pectinase.

Este trabalho propõe um método de CID miniaturizado e rápido para determinação de metanol em bebidas alcoólicas com redução considerável do consumo de reagentes e amostras.

\section{Material e métodos}

\section{Reagentes, soluções e amostras}

Neste trabalho, todos os reagentes utilizados foram de grau analítico (P.A.) e água deionizada obtida por meio de um sistema de purificação (Milli-Q, Millipore, Billerica, MA, USA) foi utilizada em todos os experimentos.

Uma solução estoque de metanol 5,0\% v/v foi preparada por diluição do reagente (Synth, São Paulo, SP, Brasil) em uma solução aquosa de etanol (Synth, São Paulo, SP, Brasil) 5,5\% v/v. Soluções de trabalho de metanol (0,01\%-0,10\% v/v) foram preparadas 
em sete níveis de concentração (em triplicatas autênticas) por diluição adequada da solução estoque em solução aquosa de etanol 5,5\% v/v. Esta mesma solução aquosa de etanol 5,5\% v/v foi utilizada como prova em branco.

Uma solução de permanganato de potássio (Synth, São Paulo, SP, Brasil) 3,0\% m/v em solução aquosa ácido fosfórico $15,0 \% \mathrm{~m} / \mathrm{v}$ foi utilizada para a oxidação do metanol, presente nas amostras, a metanal (formaldeído). Uma solução aquosa de bissulfito de sódio (Synth, São Paulo, SP, Brasil) 2,0\% m/v foi utilizada para eliminar qualquer permanganato de potássio remanescente após a oxidação do metanol presente nas amostras. Uma solução aquosa de ácido cromotrópico (Neon, Suzano, SP, Brazil) 5,0\% m/v foi utilizada como reagente cromogênico. Ácido sulfúrico concentrado (Neon, Suzano, SP, Brazil) foi utilizado para acidificação amostras e soluções padrão na etapa da reação entre o formaldeído, produzido a partir do metanol, e o ácido cromotróprico.

Uma amostra de licor de cacau (Theobroma cacao), uma amostra de licor de jambu (Acmella oleracea), uma amostra de licor de cupuaçu (Theobroma grandiflorum) e três amostras de marcas diferentes de cachaça foram adiquiridas em supermercados nos estado do Amazonas, Brasil.

Antes das análises, as amostras coloridas (licor) foram destiladas inicialmente e diluídas em água de modo que seu teor de etanol estivesse em $5,5 \% \mathrm{v} / \mathrm{v}$ enquanto que as amostras de cachaça foram diretament diluídas em água de modo a ter o mesmo teor de etanol supracitado.

\section{Equipamentos}

Um espectrofotômetro (marca Bel, modelo LGS53) utilizando um cubeta de vidro com caminho óptico de $1,0 \mathrm{~cm}$ foi utilizado para a obtenção das medidas de referência.

Um microplaca de plástico transparente contendo 96 poços (volume interno de $500 \mu \mathrm{L}$ ) de fundo chato (marca Corning®) foi utilizada para reduzir o consumo de amostras e reagentes, bem como aumentar a frequência analítica do método proposto.

Um scanner de mesa (marca Hewlett-Packard HP, modelo G2710) foi utilizado para aquisição de imagens da microplaca contendo amostras submetidas à reação cromogênica. As imagens foram capturadas com uma resolução de 300 pontos por polegada (dpi) e salvas em formato de arquivo de imagem JPG. A fim de fornecer uma iluminação uniforme sobre a microplaca, a tampa original do scanner foi substituída por outra confeccionada em madeira medindo aproximadamente $36 \times 26 \times 4 \mathrm{~cm}$ (Figura 2a). Objetivando evitar espalhamento de luz e reflexão especular, as paredes internas da nova tampa foram pintandas com tinta spray branco fosco.

\section{Programa computacional e tratamento das imagens}

O ImageJ (Figura 2b) é um programa computacional que foi escrito em liguagem Java a fim de permitir o processamento de imagens (Gonzales e Wood, 2009). Trata-se de um programa de domínio público que pode ser baixado diretamente no sítio https://imagej.nih.gov/ij/.

A rotina de operação para o tratamento de imagens pelo Image J é a seguinte: Inicialmente o usuário define no arquivo de imagem a ser analisado uma região de interesse (RI) medindo 45 x 45 elementos de imagem (pixels) no poço da microplaca que está relacionado à prova em branco. 0 programa então realiza uma varredura (coluna por coluna) de todos os pixels da RI e obtem os valores individuais de cada pixel das componentes de cor vermelho (R), verde (G) e azul (B). A partir destes são obtidos os histogramas de cada componente de cor, cujos valores médios são arredondados para o valor inteiro mais próximo. 0 processo é repetido para todos os poços da microplaca que contem soluções de calibração/amostras para a obtenção de suas respectivas componentes de cor. 
(a)

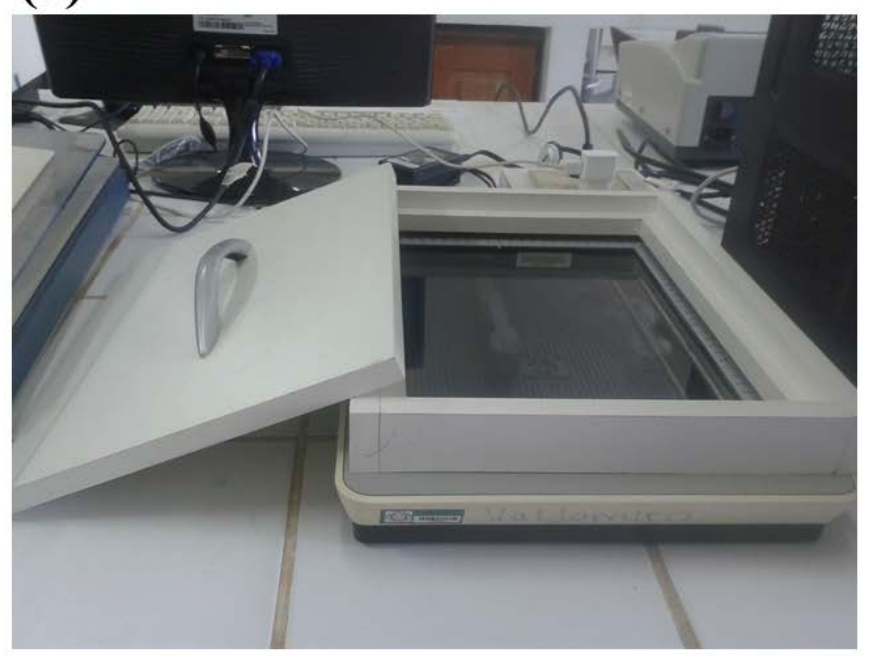

(b)

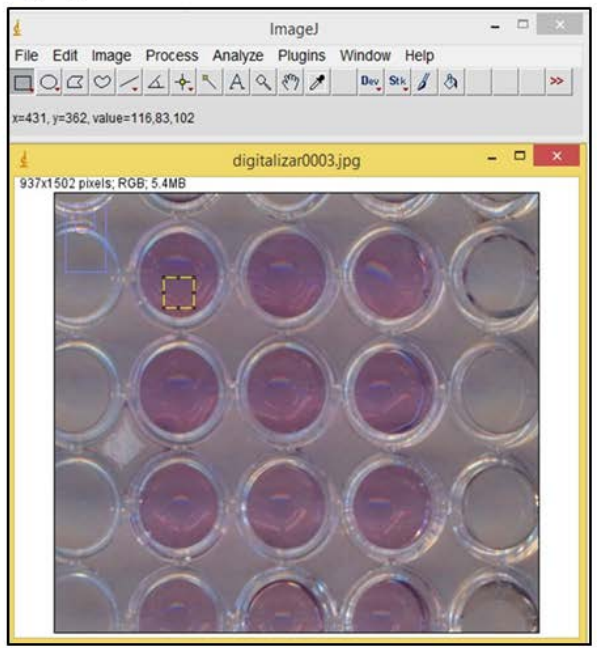

Figura 2. (a) Scanner adaptado; (b) Tela do programa ImageJ.

Índice colorimétrico de imagens digitais, curva analítica e figuras de mérito

As componentes de cor R, G e B de uma dada RI de uma dada imagem digital definem um ponto no espaço tridimensional RGB. A partir do ponto no despaço definido pelas coordenadas da prova em branco é possível definir vetores posição $\mathbf{V}$ associado às soluções padrão de metanol/amostra. Uma relação matemática entre as variações nas componentes de cor RGB é adotada como resposta analítica para construção de curvas analíticas, conforme descrito a seguir.

O índice colorimétrico de imagens digitais (ICID) é calculado como:

$$
\mathrm{ICID}=\sqrt{\overline{\mathrm{R}}_{\mathrm{b}-\mathrm{s}}^{2}+\overline{\mathrm{G}}_{\mathrm{b}-\mathrm{s}}^{2}+\overline{\mathrm{B}}_{\mathrm{b}-\mathrm{s}}^{2}}
$$

Onde: $\overline{\mathrm{R}}_{\mathrm{b} \text {-s }}^{2} \overline{\mathrm{G}}_{\mathrm{b} \text {-s }}^{2}$ e $\overline{\mathrm{B}}_{\mathrm{b} \text {-s }}^{2}$ resultam das diferenças entre os valores médios (da RI) das compoentes $\overline{\mathrm{R}}_{\mathrm{b}}, \overline{\mathrm{G}}_{\mathrm{b}}$ e $\overline{\mathrm{B}}_{\mathrm{b}}$ associadas à prova em branco e as $\overline{\mathrm{R}}_{\mathrm{s}}, \overline{\mathrm{G}}_{\mathrm{s}}$ e $\overline{\mathrm{B}}_{\mathrm{s}}$ associadas às soluções padrão/amostras.

Neste momento é necessário fazer duas considerações:

(1) A quantidade de fótons transmitidos pelas soluções, e que consequentemente alcançam o fototrandutor do scanner, é proporcional à intensidade da fonte de radiação (I).

(2) A relação entre "I" e a concentração do analito (C) para um faixa definida de concentração é linear. 
Uma vez que as duas condições supracitadas são verdadeiras, então é possível afirmar que a seguinte equação é verdadeira:

$$
\mathrm{ICID}=\mathrm{kC}
$$

Desta forma, e Equação (2) descreve a relação linear entre os valores de ICID e a concentração do analito conforme demonstrado nas seções seguintes. $\mathrm{O}$ ajuste do modelo de regressão foi verificado por meio da análise de variância (ANOVA). 0 desempenho analítico da CID foi avaliado em termos das seguintes figuras de mérito: sensibilidade, limite de detection (LD), limite de quantificação (LQ) e desvio-padrão relativo (DPR) conforme recomendações da IUPAC (1976). Uma vez que a resposta analítica é uma função de três variáveis, uma abordagem matemática deve ser usada para as estimativas do desvio-padrão da prova em branco $\left(\mathrm{s}_{\mathrm{b}}\right)$, LD e LQ. Neste caso, $\mathrm{s}_{\mathrm{b}}$ foi estimado de acordo com Lyra et al. (2009):

$$
s_{b}=\sqrt{\frac{\left(\bar{R}_{b} s_{\bar{R}}\right)^{2}+\left(\bar{G}_{b} s_{\bar{G}}\right)^{2}+\left(\bar{B}_{b} s_{\bar{B}}\right)^{2}}{\bar{R}_{b}^{2}+\bar{G}_{b}^{2}+\bar{B}_{b}^{2}}}
$$
equações:

Então, usando a Equação (3) é possivel estimar LD e LQ usando as seguintes

$$
\begin{aligned}
& \mathrm{LD}=\frac{3 \mathrm{~s}_{\mathrm{b}}}{\beta} \\
& \mathrm{LQ}=\frac{10 \mathrm{~s}_{\mathrm{b}}}{\beta}
\end{aligned}
$$

Onde " $\beta$ " é o coeficente angular da curva analítica baseada na Equação (2) e estimado por meio do ajuste de regressão pelo método dos mínimos quadrados (MMQ).

\section{Método analítico colorimétrico}

A molécula de metanol praticamente não absorve radiação na região visível do espectro eletromagnético. Tal fato inviabiliza a sua determinação direta por meio de medidas de absorbância em quaisquer comprimentos da supracitada região, independente do tipo de matriz analítica envolvida. Em situações como esta, a alternativa é converter o analito numa outra espécie química que seja facilmente detectada por uma dada técnica analítica (derivatização). Tal procedimento pode ter tantas etapas quanto necessário para uma detecção conveniente.

Para a determinação de metanol é sempre necessário um procedimento de derivatização. Entre tais procedimentos, a oxidação do metanol a formaldeído é o mais adequado. Entre os agentes oxidantes descritos na literatura, o permanganato de potássio 
em meio ácido aparece com uma opção barata, simples e rápida. A reação iônica líquida balanceada da derivatização do metanol é mostrada na Figura 3a.

0 formaldeído, assim como o metanol, também não absorve radiação na região visível do espectro eletromagnético e assim mais uma etapa de derivatização se faz necessária para a determinação de metanol. A literatura tambem descreve varios procedimentos para derivatização do formaldeído. Entre estes, a reação com a ácido cromotrópico em meio fortemente ácido é a melhor opção devido à sua rapidez, simplicidade, precisão, repetitividade, reprodutibilidade e relativo baixo custo (Fagnani et al., 2009). Embora o mecanismo e os produtos desta reação ainda sejam objeto de discussões, a proposta de mecanismo e produtos de reação mais aceita é a formulada por Georghiou e Ho (1989) (Figura 3b) cujo produto da reação absorve radiação eletromagnética entre 570-580 $\mathrm{nm}$.

(a)

$$
2 \mathrm{MnO}_{4}^{-}{ }_{(\mathrm{aq})}^{-}+5 \mathrm{CH}_{3} \mathrm{OH}_{(\mathrm{aq})}+6 \mathrm{H}_{(\mathrm{aq})}^{+} \rightarrow 5 \mathrm{CH}_{2} \mathrm{O}_{(\mathrm{aq})}+2 \mathrm{Mn}_{(\mathrm{aq})}^{+2}+8 \mathrm{H}_{2} \mathrm{O}_{(\mathrm{l})}
$$

(b)<smiles>C=CC(C)(C)Oc1ccc(O)c2c1cc(S(=O)(=O)O)c1cc3c(S(=O)(=O)O)cc4c(S(=O)(=O)O)ccc(O)c4c3[o+]c12</smiles>

Figura 3. (a) Reação global iônica de oxidação do metanol a formaldeído pelo permanganato de potássio em meio ácido; (b) Reação entre o formaldeído e ácido cromotrópico em meio fortemente ácido (não balanceada).

\section{Procedimento analítico}

Oxidação do metanol a formaldeído: Em um tubo de ensaio foram colocados $250 \mu \mathrm{L}$ da solução de permanganato de potássio e $125 \mu \mathrm{L}$ da solução padrão de metanol/amostra. Essa mistura inical foi colocada em um banho de gelo por 20 min. Após este período, quealquer eventual excesso de permanganato de potássio foi eliminado mediante a adição da solução de bissulfito de sódio (3-4 gotas).

Reação cromogênica (derivatização do formaldeído): No mesmo tubo de ensaio após a adição do bissulfito de sódio, foram adicionados $250 \mu \mathrm{L}$ da solução de ácido cromotrópico e 1,9 mL de ácido sulfúrico concentrado. Esta nova mistura foi então colocada em banho-maria $\left(60^{\circ} \mathrm{C}-75^{\circ} \mathrm{C}\right)$ por $15 \mathrm{~min}$. A solução final foi resfriada até temperatura ambiente e o volume da mesma foi levado a $10 \mathrm{~mL}$ em balão volumétrico usando água deionizada. Uma prova em branco foi preparada seguindo todo o procedimento, mas usando água deionizada no lugar da solução padrão de metanol/amostra. As soluções resultantes referentes à prova em branco, soluções padrão de metanol e amostras foram colocadas nos poços da microplaca para aquisição das imagens $(350 \mu \mathrm{L})$. Estas mesmas soluções foram utilizadas nas medidas de absorbância em $575 \mathrm{~nm}$ para obtenção dos valores de referência das amostras. 


\section{Resultados and discussão}

\section{Imgens digitais e o fenômeno de absorção de radiação eletromagnética}

A Figura 4a mostra a imagem da microplcac contendo o produto da reação entre formaldeído e ácido cromotrópico referente as sete concentrações de metanol. 0 primeiro poço refere-se à prova em branco (quando o analito está ausente). Como pode ser visto, os poços apresentam a cor púrpura (marcadamente nos poços 6 e 7) para qual a intensidade aumenta à medida que a concentração de metanol nas soluções padrão aumenta. Esta radiação emergente (transmitida) corresponde à absorção de radiação eletromagnética na região amarelo-esverdeado (560-580 nm) (Jeffery et al., 1989).

De fato, ao observar como as componentes RGB variam à medida que a concentração de metanol nas soluções padrão aumenta (Figura 4b) é possível constatar seguinte ordem de dominância na contribuição da cor nos poços: $G$ >> R > B.

(a)

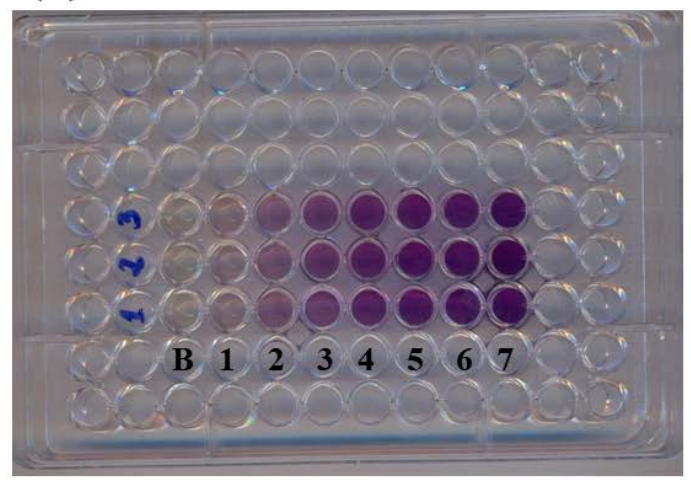

(b)

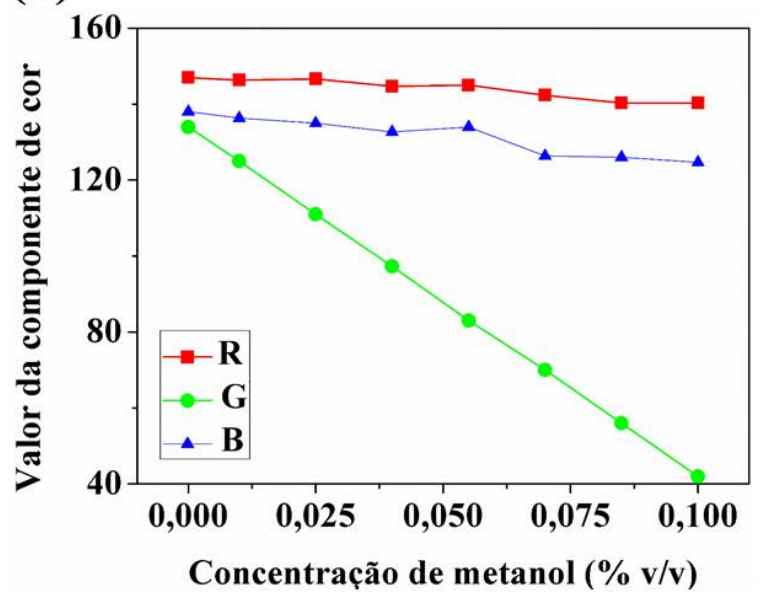

Figura 4. (a) Microplaca contendo o produto de reação referente a cada solução padrão de metanol. (b) Variação das componentes RGB em função do aumento da concentração de metanol.

\section{Otimização das condições experimentais}

Quatro fatores foram avalidados de modo a aumentar a reprodutividade das medidas, bem como a sensibilidade da resposta analítica: posição da microplaca no scanner, tamanho da RI, quantidade de varreduras do scanner e volume de solução no poço da microplaca. Para tal, foi utilizada uma solução padrão de $0,1 \% \mathrm{v} / \mathrm{v}$ de metanol a qual foi submetida a todo o procedimento analítico em três poços vizinhos. 0 menor desvio padrão (DP) absoluto $(n=10)$ no valor de ICID é o critério de escolha para uma dado nível de um dado fator. Os resultados são apresentados na Tabela 1.

A influência da posição da microplaca no scanner foi avaliada de modo a evitar a reflexão de luz nas bordas do poço. Seis posições diferentes (Figura 5a1-a6) foram avaliadas. Destas, a que apresentou menor reflexão de luz na bordas dos poços foi a posição (a5). 
Tabela 1. Valores de DP para os seis níveis dos quatro fatores avalidos.

\begin{tabular}{lcccccc}
\hline Fatores & \multicolumn{7}{c}{ Níveis } \\
\hline Posição da microplaca* & $(\mathrm{a} 1)$ & $(\mathrm{a} 2)$ & $(\mathrm{a} 3)$ & $(\mathrm{a} 4)$ & $(\mathrm{a} 5)$ & $(\mathrm{a} 6)$ \\
DP & 1,46 & 1,26 & 1,46 & 0,98 & 0,78 & 1,13 \\
\hline Tamanho da RI* & $(\mathrm{b} 1)$ & $(\mathrm{b} 2)$ & $(\mathrm{b} 3)$ & $(\mathrm{b} 4)$ & $(\mathrm{b} 5)$ & $(\mathrm{b} 6)$ \\
DP & 0,56 & 1,06 & 0,90 & 1,07 & 1,07 & 1,06 \\
\hline Varreduras do scanner & 1 & 2 & 3 & 6 & 9 & 12 \\
DP & 1,48 & 1,06 & 0,77 & 0,77 & 0,78 & 0,78 \\
\hline Volume de solução $(\mu \mathrm{L})$ & 150 & 200 & 250 & 300 & 350 & 400 \\
DP & 0,94 & 0,84 & 0,70 & 0,63 & 0,56 & 1,04 \\
\hline
\end{tabular}

*Níveis descritos na Figura 5.

A influência do tamanho da RI foi avaliada de modo a verificar em qual região do poço da microplaca a cor se mostrava mais uniforme. Destas, as regiões (b2), (b4), (b5) e (b6) apresentaram menor uniformidade por se situarem próximas às bordas dos poços da microplaca. Apesar se se situarem no centro do poço da microplaca, as regiões (b1) e (b3) apresentaram diferenças na uniformidade na cor. Isto se deve basicamente ao tamanho das áreas utilizadas. Neste caso a área da região (b1) é aproximadamente $156 \%$ maior que a da (b3).

A influência da quantidade de varreduras do scanner foi avaliada de modo a verficar a necessidade de uma estabilização incial do sensor de imagem do mesmo. De fato, as medidas se mostraram bastante estáveis a partir da terceira varredura do scanner. Sendo assim se faz necessário antes das atividades analíticas, três varredurais iniciais.

A influência do volume de solução a ser transferido para o poço da microplaca foi avaliada de modo a verificar o aumento máximo devido ao aumento do caminho ótico referente à solução. 0 valor limite encontrado foi de $350 \mu \mathrm{L}$. A partir deste valor é provável que haja o desvio da Lei de Beer.

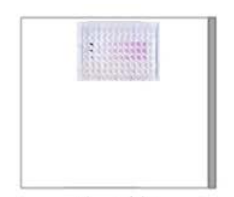

(a1)

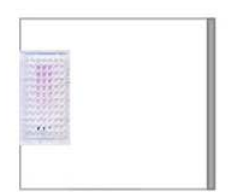

(a4)

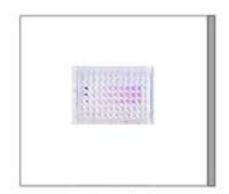

(a2)

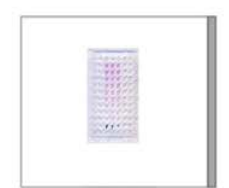

(a5)

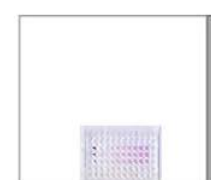

(a3)

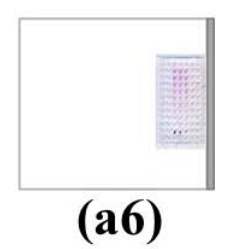

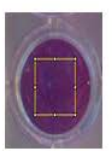

(b1) (b2)

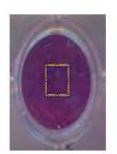

(b3) (b4)

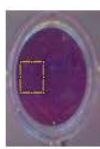

(b5) (b6)

Figura 5. (a1-a6) posições da microplaca sobre o scanner. (b) Tamanhos e posições da RI na imagem do poço da microplaca: (b1) 45 x 45 pixels no centro, (b2) 25 x 25 pixels na parte superior, (b3) 25 x 25 pixels no centro, (b4) 25 x 25 pixels na parte inferior, (b5) $25 \times 25$ pixels à esquerda, e (b6) 25 x 25 pixels à direita. 
As condições experimentais para o levantamento da curva analítica foram: microplaca na posição (a5), região da microplaca (b1), medidas realizada após três varreduras inciais e volume de solução transferido para poço da microplaca igual a $350 \mu \mathrm{L}$.

\section{Curva analítica, análise de variância e desempenho do método CID}

A Figura 6a mostra a curva analítca utilizada para determinação de metanol em amostras de bebidas alcoólicas. $\mathrm{O}$ ajuste dos dados utilizando o MMQ forneceu o seguinte modelo de primeira ordem: ICID $=(-0,1021 \pm 0,1936)+(933,02 \pm 8,4478) C$; onde C é a concentração de metanol em \% v/v. 0 coeficiente de determinação do modelo $\left(\mathrm{R}^{2}\right)$ revela que $99,99 \%$ da variância dos dados pode ser descrita pelo modelo linear.

Analisando os intervalos de confiança dos coeficientes do modelo construído (ao nível de 95\%) observou-se que para o coeficiente linear seu intervalo inclui o "zero", logo este não possui significância estatística. Em síntese: a variância dos dados da curva analítica é descrita apenas pelo coeficiente angular.

Uma ANOVA foi implementada a fim de validar a curva analítica. Para tal foram realizados dois testes $F$ : o primeiro para verificar a falta de ajuste e o segundo para verificar a significância da regressão (Draper e Smith, 1998). Para o teste de falta de ajuste, a curva analítica foi contruída com base em triplicatas autênticas em sete níveis de concentração. Os valores de erro puro e falta de ajuste foram calculados a partir das somas quadráticas (SQ) e médias quadráticas (MQ) apresentadas na Tabela 2.

Tabela 2. ANOVA para o ajuste do modelo linear $(\hat{y}=\beta X)$ da curva analítica (Figura 6a).

\begin{tabular}{lccc}
\hline Fonte & SQ & Graus de liberdade & MQ \\
\hline Regressão & $7,64 \times 10^{3}$ & 1 & $7,64 \times 10^{3}$ \\
Residual & $1,09 \times 10^{-1}$ & 19 & $5,76 \times 10^{-3}$ \\
Falta de ajuste & $3,56 \times 10^{-4}$ & 5 & $7,12 \times 10^{-5}$ \\
Erro puro & $1,09 \times 10^{-1}$ & 14 & $7,80 \times 10^{-3}$ \\
\hline
\end{tabular}

$\mathrm{O}$ teste $F$ para falta de ajuste consiste em comparar a razão $\mathrm{MQ}_{\text {falta de ajuste }} / \mathrm{MQ}_{\text {erro puro }}$ com o valor do ponto da distribuição $F$, considerando os mesmos graus de liberdade das respectivas MQ's a um dado nível de confiança (neste trabalho, 95\%). Neste estudo, esta razão foi de $9,14 \times 10^{-3}$ enquanto que o ponto da distribuição $F$, considerando os mesmos graus de liberdade $\left(F_{5,14} 95 \%\right)$, é 2,958. Uma fez que $F_{\text {calc }}<F_{\text {tab, }}$ pode-se afirmar que não há evidências de erros sistemáticos na construção da curva analítica. De fato, o gráfico de resíduos deixado pelo modelo (Figura 6b) mostram uma distribuição aleatória em torno de zero, corroborando o resultado anterior.

0 teste $F$ para significância de regressão consiste em comparar a razão $M Q_{\text {regressão }} / M_{\text {residual }}$ com o valor do ponto da distribuição $F$ (como na falta de ajuste). Neste estudo, esta razão foi de $1,33 \times 10^{6}$ enquanto que o ponto da distribuição $F$, considerando os mesmos graus de liberdade $\left(F_{1,19} 95 \%\right)$, é 4,381 . Uma fez que $F_{\text {calc }}>F_{\text {tab, }}$, pode-se afirmar que a regressão é altamente significativa.

Uma vez que os resultados da ANOVA demonstram que não há falta de ajuste e que há alta significância na regressão, pode-se afirma que a curva de calibração segue um modelo linear e validado.

Após a validação da curva analítica, as figuras de mérito puderam ser estimadas. Como pode ser visto o método CID produziu uma curva analítica com uma considerável sensibilidade, que pode ser atribuída à natureza trivariada da resposta analítica (Lyra et 
al., 2009). Foram utilizadas 30 varreduras da prova em branco para estimar os valores de LD e LQ os quais foram $1,95 \times 10^{-3}$ e $6,50 \times 10^{-3} \% \mathrm{v} / \mathrm{v}$, respectivamente.

(a)

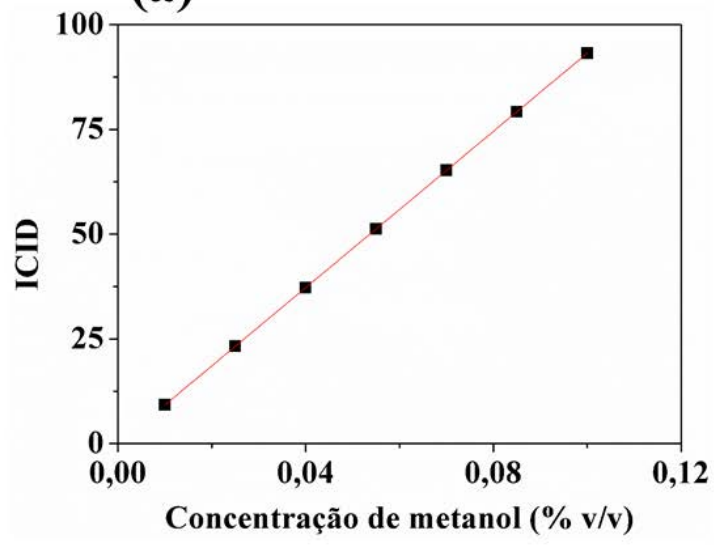

(b)

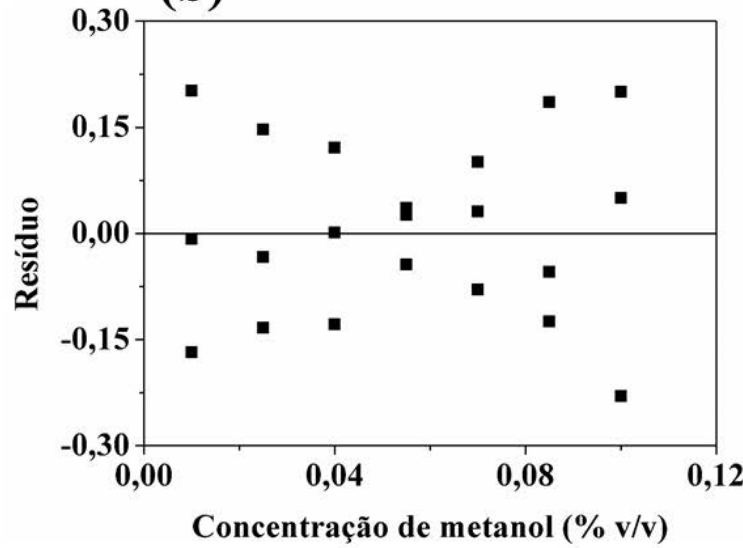

Figura 6. (a) Curva analítica obtida utilizando as condições otimizadas na seção anterior. (b) Gráfico de resíduos do modelo linear usado para construção da curva analítica.

Adimitindo que o tempo para aquisição de uma imagem de uma microplaca (considerando a troca de amostras) é de 30 s e considerando uma microplaca de 96 poços, o método proposto é capaz de analizar até 11.520 amostras/h.

\section{Determinações analíticas}

De acordo com a Tabela 3, a CID e a espectrometria UV-Vis produziram resultados similares na determinação de metanol em bebidas alcoólicas. De fato, nenhuma diferença estatísticamente significativa foi verificada entre os resultados ao aplicar o teste- $t$ emparelhado ao nível de confiança de 95\%. Entretanto a CID apresentou melhor precisão, conforme revelado pelo menor valor de desvio padrão relativo (DPR) conjunto. Esta precisão é atribuída à natureza trivairada da resposta analítica (ICID).

Tabela 3. Resultados da determinação de metanol (\% v/v) em bebidas alcoólicas (n = 3).

\begin{tabular}{lcc}
\hline Amostra & CID & UV-Vis \\
\hline Cachaça 1 & $0,0270 \pm 0,0008$ & $0,0283 \pm 0,0009$ \\
Cachaça 2 & $0,0216 \pm 0,0001$ & $0,0209 \pm 0,0013$ \\
Cachaça 3 & $0,0373 \pm 0,0006$ & $0,0375 \pm 0,0018$ \\
Licor de jambu & $0,0150 \pm 0,0006$ & $0,0143 \pm 0,0016$ \\
Licor de cupuaçu & $0,0219 \pm 0,0007$ & $0,0222 \pm 0,0007$ \\
Licor de cacau & $0,0190 \pm 0,0007$ & $0,0186 \pm 0,0010$ \\
\hline DPR conjunto $\%)$ & 2,67 & 5,38 \\
\hline
\end{tabular}

\section{Conclusão}

Este trabalho utiliza a aquisição de imagens digitais mediant um scanner para implementar um método CID para determinação de metanol em bebidas alcoólicas. 0 uso de um scanner, cujo custo de aquisição e manutenção é relativamente baixo, como 
detector analítico é uma alternativa rápida, barata, confiável e atraente para a tradional espectrometria UV-Vis. O método CID proposto apresentou desempenho satisfatório quando comparado com o mesmo aplicado em outras técnicas analíticas (Tabela 4).

0 incremento significativo na frequência analítica é atribuído à resolução espacial inerente às imagens digitais. Além disso o uso da microplaca possibilita a redução do consumo de reagente e amostras, bem como a redução na geração de resíduos químicos como preconizados pelos princípios da química analítica verde.

Tabela 4. Características analíticas do método CID e outras técnicas analíticas para determinação de metanol em bebidas alcoólicas.

\begin{tabular}{lccc}
\hline Parâmetro & CID & UV-Vis & $\begin{array}{c}\text { DIB spot test } \\
\text { (Franco et al., 2016) }^{\mathbf{a}}\end{array}$ \\
\hline Detector & Scanner & Espectrofotométrico & Câmera digital \\
LD (\% v/v) & 0,0019 & 0,0032 & 0,0019 \\
Faixa de trabalho LD (\% v/v) & $0,0100-0,1000$ & $0,0100-0,0800$ & $0,0063-0,1842$ \\
Sensibilidade de calibração (1/(\% v/v)) & 933,0200 & 8,5851 & 12,7050 \\
Frequência analítica (amostras/hora) & $11520^{\mathrm{b}}$ & $360^{\mathrm{c}}$ & $5400^{\mathrm{d}}$ \\
Consumo de reagentes (mL) & 9,875 & 9,875 & 0,720 \\
Consumo de amostra ( $\mathrm{QL})$ & 125 & 125 & 80 \\
\hline
\end{tabular}

a As unidades descritas no trabalho (mg/100 mL) foram convetidas para fins de comparação.

b base de cálculo: $30 \mathrm{~s}$ para aquisição de imagem e troca da microplaca (96 poços).

c base de cálculo: $10 \mathrm{~s}$ para medida e troca de amostra na cubeta.

d base de cálculo: $6 \mathrm{~s}$ para aquisição de imagem e troca da placa de spot test ( 9 poços).

\section{Agradecimentos}

Os autores agradecem à Fundação de Amparo a Pesquisa do Estado do Amazonas (FAPEAM) pela bolsa de mestrado concedida à primeira autora.

\section{Conflito de interesses}

Os autores declaram não haver conflito de interesses.

\section{Referências}

Byrne, L.; Barker, J.; Pennarun-Thomas, G.; Diamond, D. Digital imaging as a detector for generic analytical measurements. Trends in Analytical Chemistry, v. 19, n. 8, p. 517-522, 2000. https://doi.org/10.1016/S0165-9936(00)00019-4

Canteri, M. H. G.; Moreno, L.; Woslocke, G.; Scheer, A, P. Pectina: da matéria-prima ao produto final. Polímeros, v. 22, n. 2, p. 149-157, 2012. https://doi.org/10.1590/S010414282012005000024

Caruso, M. S. F.; Nagato, L. A. F.; Alaburda, J. Benzo(a)pireno, carbamato de etila e metanol em cachaças. Química Nova, v. 33, n. 9, p. 1973-1976, 2010. https://doi.org/10.1590/ S0100-40422010000900027

Draper, N. R.; Smith, H. Applied regression analysis. 3. ed. New York: Wiley, 1998. 
Fagnani, E.; Pezza, L.; Pezza, H. R. Determinação espectrofotométrica de hexametilenotetramina (HMT) em medicamentos, utilizando ácido cromotrópico e forno de micro-ondas. Química Nova, v. 32, n. 9, p. 2458-2463, 2009. https://doi.org/ 10.1590/S0100-40422009000900039

Ferreira, E. C.; Montes, R. A Química da produção de bebidas alcoólicas. Química Nova na Escola, n. 10, p. 50-51, 1999.

Franco, M. K.; Suarez, W. T.; Maia, M. V.; Santos, V. B. Smartphone application for methanol determination in sugar cane spirits employing digital image-based method. Food Analytical Methods, v. 10, n. 6, p. 2102-2109. https://doi.org/10.1007/s12161-0160777-y

Geladi, P.; Grahn, H.; Esbensen, K.; Bengtsson, E. Image analysis in chemistry II. Multivariate image analysis. Trends in Analytical Chemistry, v. 11, n. 3, p. 121-130, 1992. https://doi.org/10.1016/0165-9936(92)85010-3

Georghiou, P. E.; Ho, C. K. The chemistry of the chromotropic acid method for the analysis of formaldehyde. Canadian Journal of Chemistry, v. 67, n. 5, p. 871-876, 1989. https://doi.org/10.1139/v89-135

Gomes, M. S.; Trevizan, L. C.; Nóbrega, J. A.; Kamogawa, M. Y. Uso de scanner em espectrofotometria de absorção molecular: aplicação em experimento didático enfocando a determinação de ácido ascórbico. Química Nova, v. 31, n. 6, p. 1577-1581, 2008. https://doi.org/10.1590/S0100-40422008000600050

Gonzales, R. F.; Woods, R. E. Processamento digital de imagens. 3. ed. São Paulo: Pearson, 2009.

IUPAC - International Union of Pure and Applied Chemistry. Nomenclature, symbols, units and their usage in spectrochemical analysis - II. Data interpretation. Pure and Applied Chemistry, v. 45, n. 2, p. 99-103, 1976. https://doi.org/10.1351/pac197645020099

Jeffery, G. H.; Basset, J.; Mendham, J.; Denney, R. C. Vogel's textbook of quantitative chemical analysis. 5. ed. London: Longman Scientific and Technical, 1989.

Lopez-Molinero, A.; Liñan, D.; Sipiera, D.; Falcon, R. Chemometric interpretation of digital image colorimetry. Application for titanium determination in plastics. Microchemical Journal, v. 96, n. 2, p. 380-385, 2010. https://doi.org/10.1016/j.microc.2010.06.013

Lyra, W. S.; Santos, V. B.; Dionízio, A. G. G.; Martins, V. L.; Almeida, L. F.; Gaião, E. N.; Diniz, P. H. G. D.; Silva, E. C.; Araújo, M. C. U. Digital image-based flame emission spectrometry. Talanta, v. 77, n. 5, p. 1584-1589, 2009. https://doi.org/10.1016/j.talanta.2008.09.057

Martins, A. R.; Paciornik, S.; Pereira, J. A. S. Brinell hardness: Image analysis and uncertainty. Proceedings of IMEKO 2000, ÖGMA - The Austrian Society for Measurement and Automation, Wien, 2000.

Ohimain, E. I. Methanol contamination in traditionally fermented alcoholic beverages: The microbial dimension. Springer Plus, v. 5, n. 1607, p. 1-10, 2016. https://doi.org/10.1186/ s40064-016-3303-1

Paciornik, S.; Yallouz, A. V.; Campos, R. C.; Gannerman, D. Scanner image analysis in the quantification of mercury using spot-tests. Journal of the Brazilian Chemical Society, v. 17, n. 1, p. 156-161, 2006. https://doi.org/10.1590/S0103-50532006000100022

Péres, L. O.; Li, R. W. C.; Yamauchi, E. Y.; Lippi, R.; Gruber, J. Conductive polymer gas sensor for quantitative detection of methanol in Brazilian sugar-cane spirit. Food Chemistry, v. 130, n. 4, p. 1105-1107, 2012. https://doi.org/10.1016/j.foodchem.2011.08.014

Rev. Bras. Gest. Amb. Sustent., 2020, vol. 7, n. 16, p. 455-468. 
Prats-Montalbán, J. M.; Juan, A.; Ferrer, A. Multivariate image analysis: A review with applications. Chemometrics and Intelligent Laboratory Systems, v. 107, n. 1, p. 1-23, 2011. https://doi.org/10.1016/j.chemolab.2011.03.002

Santos, P. M.; Wentzel, P. D.; Pereira-Filho, E. R. Scanner digital images combined with color parameters: A case study to detect adulterations in liquid cow's milk. Food Analytical Methods, v. 5, n. 1, p. 89-95. https://doi.org/10.1007/s12161-011-9216-2

Seyfried, M.; Soldera-Silva, A.; Bovo, f.; Stevan-Hancke, F. R.; Maurer, J. B. B.; ZawadzkiBaggio, S. F. Pectinas de plantas medicinais: características estruturais e atividades imunomoduladoras. Revista Brasileira de Plantas Medicinais, v. 18, n. 1, p. 201-214, 2016. https://doi.org/10.1590/1983-084X/15_078

Soldat, D. J.; Barak, P.; Lepore, B. J. Microscale colorimetric analysis using a desktop scanner and automated digital image analysis. Journal of Chamical Education, v. 48, n. 5, p. 617-620, 2009. https://doi.org/10.1021/ed086p617

Soponar, F.; Moț, A. C.; Sârbu, C. Quantitative determination of some food dyes using digital processing of images obtained by thin-layer chromatography. Journal of Chromatography A, v. 1188, n. 2, p. 295-300, 2008. https://doi.org/10.1016/j.chroma. 2008.02.077

Zacaroni, L. M.; Cardoso, M. G.; Saczk, A. A.; Santiago, W. D.; Anjos, J. P.; Masson. J.; Duarte, F. C.; Nelson, D. L. Caracterização e quantificação de contaminantes em aguardentes de cana. Química Nova, v. 34, n. 2, p. 320-324, 2011. https://doi.org/10.1590/S010040422011000200026

Informação da Licença: Este é um artigo Open Access distribuído sob os termos da Licença Creative Commons Attribution, que permite uso irrestrito, distribuição e reprodução em qualquer meio, desde que a obra original seja devidamente citada. 